\title{
The Cube Attack on Stream Cipher Trivium and Quadraticity Tests*
}

\author{
Piotr Mroczkowski and Janusz Szmidt \\ Military Communication Institute \\ ul. Warszawska 22A, 05-130 Zegrze, Poland \\ Military University of Technology \\ ul. Kaliskiego 2, 00-980 Warsaw, Poland
}

November 14, 2010

\begin{abstract}
In 2008 I. Dinur and A. Shamir presented a new type of algebraic attack on symmetric ciphers named cube attack. The method has been applied to reduced variants of stream ciphers Trivium and Grain128, reduced variants of the block ciphers Serpent and CTC and to a reduced version of the keyed hash function MD6. Independently a very similar attack named AIDA was introduced by M. Vielhaber. In this paper we develop quadraticity tests within the cube attack and apply them to a variant of stream cipher Trivium reduced to 709 initialization rounds. Using this method we obtain the full 80-bit secret key. In this way it eliminates the stage of brute force search of some secret key bits which occured in previous cube attacks.
\end{abstract}

\section{Introduction}

The cube attack has been introduced by Itai Dinur and Adi Shamir [8] as a known plaintext attack on symmetric primitives. The method has been further developed in $[2,3,9,10]$. The ciphertext bits produced by this algorithm are values of polynomials $p\left(v_{1}, \ldots, v_{m}, x_{1}, \ldots, x_{n}\right)$ depending on public variables $v_{1}, \ldots, v_{m}$ (bits of a plaintext for block ciphers or bits of an initial vector for stream ciphers) and depending on secret variables $x_{1}, \ldots, x_{n}$ (bits of a key). The attack consists of two stages. In the first preprocessing stage the attacker has access to public and secret variables. He sums up ciphertext bits obtained for chosen $k$-dimensional cubes in public variables

*This papre was presented at the 10th Central European Conference on Cryptology in Będlewo, Poland. 
and fixed key variables. The attacker chooses different keys and obtains a function depending on key bits. The task of this stage is to find the cases where this function is affine or quadratic and reconstruct it. The tools to investigate the linearity or quadraticity of the resulting Boolean functions are the linearity and quadraticity tests, developed in $[1,5]$. The preprocessing stage is the most time consuming part of the attack. Heuristic considerations and experiments are involved to find suitable cubes.

In the next on line stage of the attack a key is secret and the attacker only has access to public variables. He sums up over the same cubes as in the preprocessing stage to obtain the right hand sides of linear and quadratic equations. Having the system of all equations the attacker tries to solve it to get values of some key bits. To solve the resulting system of quadratic equations, linearization methods can be applied. The remaining, unknown bits of the key can be calculated by brute force searching. Dinur and Shamir [8] applied the cube attack to variants of stream cipher Trivium reduced to 672,735 and 767 initialization rounds (the whole cipher runs over $4 \times 288=$ 1152 initial rounds before producing output key bits). They used linearity tests and obtained a system of linear equations for key bits. In the case of 767 initialization rounds they obtained 35 linear expressions for key bits. The remaining $80-35=45$ bits of the secret key dominate the complexity of an attack being $O\left(2^{45}\right)$ cipher executions which is below the complexity $O\left(2^{80}\right)$ of the brute force search of all key bits. In [8] it was suggested to apply quadraticity tests within the cube attack.

We realize this idea and obtain quadratic and linear expressions involving key bits for a variant of Trivium reduced to 709 initialization rounds. Quadratic terms appear more often than linear ones and this leads to more equations for key bits. In our attack we used 22 and 23 dimensional cubes. We found 41 bits of a randomly chosen secret key using linear terms and 39 remaining bits of this key using quadratic equations. In fact, quadratic equations were solved by hand: substituting bits obtained from linear equations and doing some manipulations. This way the brute force searching of some bits of the secret key was completely eliminated. The complexity of the on line stage of our cube attack is about $2^{29}$ executions of reduced Trivium with $709 \div 713$ initialization rounds. The system of linear and quadratic expressions obtained during the preprocessing stage can be used to find any secret key.

It is important to have an effective implementation of the cipher in question to perform the cube attack. We used Paul Crowley's [7] implementation of Trivium, which was written using assembly CorePy tool. In this case 128 parallel strings of output key bits are prduced on the level of processor instructions. These strings are used to perform summation over chosen cubes and speed up the preprocessing stage of the attack. 


\section{Linearity and Quadraticity Tests}

Let $F_{2}^{n}$ be the $n$-dimensional vector space over the binary field $F_{2}$ and $f$ : $F_{2}^{n} \longrightarrow F_{2}$ a Boolean function of $n$ binary variables. The Boolean function $f$ is affine if it satisfies the linearity test:

$$
f\left(x \oplus x^{\prime}\right)=f(x) \oplus f\left(x^{\prime}\right) \oplus f(0)
$$

for all $x, x^{\prime} \in F_{2}^{n}$. Such a function has the following Algebraic Normal Form (ANF):

$$
f\left(x_{1}, \ldots, x_{n}\right)=\bigoplus_{1 \leq i \leq n} a_{i} x_{i} \oplus a_{0},
$$

where $a_{0}, a_{1}, \ldots, a_{n}$ are binary coefficients.

The Boolean function $f$ is quadratic if it satisfies the quadraticity test:

$$
\begin{gathered}
f\left(x \oplus x^{\prime} \oplus x^{\prime \prime}\right)=f\left(x \oplus x^{\prime}\right) \oplus f\left(x \oplus x^{\prime \prime}\right) \oplus f\left(x^{\prime} \oplus x^{\prime \prime}\right) \\
\oplus f(x) \oplus f\left(x^{\prime}\right) \oplus f\left(x^{\prime \prime}\right) \oplus f(0)
\end{gathered}
$$

for all $x, x^{\prime}, x^{\prime \prime} \in F_{2}^{n}$. Such a function has the following ANF form:

$$
f\left(x_{1}, \ldots, x_{n}\right)=\bigoplus_{1 \leq i<j \leq n} a_{i j} x_{i} x_{j} \oplus \bigoplus_{1 \leq i \leq n} a_{i} x_{i} \oplus a_{0}
$$

for some binary coefficients $a_{i j}, a_{i}, a_{0}$.

Let us note that a function satisfying the linearity test also satisfies the quadraticity test, with all $a_{i j}$ equal to zero. The main phenomenon appearing in the cube attack is that quadratic or affine Boolean functions can be detected with high probability by executing the quadraticity or linearity tests only for a very small number of triples $\left(x, x^{\prime}, x^{\prime \prime}\right)$ or pairs $\left(x, x^{\prime}\right)$. Linearity tests and their information-theoretical applications were investigated by Blum, Luby and Ribenfeld [5]. Testing of low-degree polynomials over $F_{2}$ was developed by Alon, Kaufman, Krivelevich, Litsyn and Ron in [1].

The coefficients in the Algebraic Normal Form can be calculated by summing over suitable cubes. The task of the preprocessing stage is to collect, for the cipher under study, as many quadratic and linear expressions in key bits as possible.

\section{Specification of Trivium}

\subsection{Key Stream Generation}

The stream cipher Trivium contains a 288-bit inner state consisting of three registers of lengths 84,93 and 111 . The key stream generation is an iterative process which extracts the values of 15 specific state bits and uses them to update 3 bits of the state and to compute 1 bit of the key stream. The 
state bits are then rotated and the process repeats. The generation of the ouput bitstring $\left(z_{i}\right)$ of the maximal length of up to $N=2^{64}$ bits, can be represented as follows:

for $\mathrm{i}=1$ to $\mathrm{N}$

$$
\begin{gathered}
t_{1} \leftarrow s_{66}+s_{93} \\
t_{2} \leftarrow s_{162}+s_{177} \\
t_{3} \leftarrow s_{243}+s_{288} \\
z_{i} \leftarrow t_{1}+t_{2}+t_{3} \\
t_{1} \leftarrow t_{1}+s_{91} \cdot s_{92}+s_{171} \\
t_{2} \leftarrow t_{2}+s_{175} \cdot s_{176}+s_{264} \\
t_{3} \leftarrow t_{3}+s_{286} \cdot s_{287}+s_{69} \\
\left(s_{1}, s_{2}, \ldots, s_{93}\right) \leftarrow\left(t_{3}, s_{1}, \ldots, s_{92}\right) \\
\left(s_{94}, s_{95}, \ldots, s_{177}\right) \leftarrow\left(t_{1}, s_{94}, \ldots, s_{176}\right) \\
\left(s_{178}, s_{179}, \ldots, s_{288}\right) \leftarrow\left(t_{2}, s_{178}, \ldots, s_{287}\right)
\end{gathered}
$$

end for

\subsection{Key and Initial Value Setup}

The 288-bit inner state of Trivium is initialized in the following way:

$$
\begin{gathered}
\left(s_{1}, s_{2}, \ldots, s_{93}\right) \leftarrow\left(k_{1}, k_{2}, \ldots, k_{80}, 0, \ldots, 0\right) \\
\left(s_{94}, s_{95}, \ldots, s_{177}\right) \leftarrow\left(I V_{1}, I V_{2}, \ldots, I V_{80}, 0, \ldots, 0\right) \\
\left(s_{178}, s_{179}, \ldots, s_{288}\right) \leftarrow(0,0, \ldots, 0,1,1,1)
\end{gathered}
$$

for $\mathrm{i}=1$ to 1152

$$
\begin{gathered}
t_{1} \leftarrow s_{66}+s_{93} \\
t_{2} \leftarrow s_{162}+s_{177} \\
t_{3} \leftarrow s_{243}+s_{288} \\
t_{1} \leftarrow t_{1}+s_{91} \cdot s_{92}+s_{171} \\
t_{2} \leftarrow t_{2}+s_{175} \cdot s_{176}+s_{264} \\
t_{3} \leftarrow t_{3}+s_{286} \cdot s_{287}+s_{69} \\
\left(s_{1}, s_{2}, \ldots, s_{93}\right) \leftarrow\left(t_{3}, s_{1}, \ldots, s_{92}\right) \\
\left(s_{94}, s_{95}, \ldots, s_{177}\right) \leftarrow\left(t_{1}, s_{94}, \ldots, s_{176}\right) \\
\left(s_{178}, s_{179}, \ldots, s_{288}\right) \leftarrow\left(t_{2}, s_{178}, \ldots, s_{287}\right)
\end{gathered}
$$

end for 


\section{Results of the Cube Attack}

\subsection{Linear Expressions}

In the preprocessing stage we found linear expressions involving unknown key bits. These expressions were obtained by summing up over cubes, which were chosen among initial value variables. 240 linearity tests were performed for each cube from Table 1 . The dimensions of the cubes (22 and 23) were fixed experimentally. In fact, the cubes were chosen randomly but also we found a method to obtain a new cube from another one with a linear expression: one must increase the indices of a cube by one (if possible) and increase the number of initialization rounds by one.

Table 1. Linear expressions.

\begin{tabular}{|c|c|c|}
\hline expression & cube indices & round \\
\hline $\mathrm{x} 14$ & 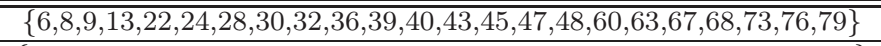 & 709 \\
\hline $\mathrm{x} 15$ & $\{2,9,15,17,27,28,32,40,44,46,52,54,59,64,68,70,71,72,73,74,76,78,79\}$ & 709 \\
\hline $\mathrm{x} 16+1$ & $\{1,3,9,10,13,14,16,28,34,37,42,51,52,56,59,60,62,68,69,72,74,79\}$ & 709 \\
\hline $\mathrm{x} 17$ & $\{4,5,6,10,11,12,17,19,21,26,32,40,44,49,54,58,60,61,67,72,74,77,78\}$ & 709 \\
\hline $\mathrm{x} 18$ & $\{5,9,15,16,20,21,32,33,35,38,41,43,46,52,56,58,60,61,62,69,77,78,79\}$ & 709 \\
\hline $\mathrm{x} 19+1$ & $\{6,8,13,17,23,27,28,33,44,45,46,53,54,56,60,61,67,68,72,74,75,77,79\}$ & 709 \\
\hline $\mathrm{x} 20$ & $\{1,2,7,13,15,18,19,23,29,34,35,36,38,47,49,54,57,62,64,65,66,68,74\}$ & 709 \\
\hline $\mathrm{x} 21$ & $\{4,7,10,11,19,20,22,23,24,30,32,33,38,41,49,52,54,59,66,67,69,74,77\}$ & 709 \\
\hline $\mathrm{x} 22$ & $\{8,16,18,22,24,26,29,31,34,36,40,41,45,46,47,48,50,59,63,69,72,76,78\}$ & 709 \\
\hline $\mathrm{x} 23+1$ & $\{6,10,13,16,19,25,28,35,39,42,44,48,57,61,62,63,64,65,67,68,73,77,78\}$ & 709 \\
\hline $\mathrm{x} 24$ & $\{2,4,7,15,17,18,20,23,24,27,29,35,45,47,48,51,57,59,63,65,67,74,77\}$ & 709 \\
\hline $\mathrm{x} 25$ & $\{3,5,8,16,18,19,21,24,25,28,30,36,46,48,49,52,58,60,64,66,68,75,78\}$ & 710 \\
\hline $\mathrm{x} 33+1$ & $\{5,10,13,14,15,22,26,27,32,35,36,45,46,50,51,56,59,60,63,64,77,78,79\}$ & 709 \\
\hline $\mathrm{x} 35+1$ & $\{2,6,8,9,19,23,24,29,32,33,34,42,47,49,51,52,53,57,61,64,73,77\}$ & 710 \\
\hline $\mathrm{x} 39$ & $\{0,3,6,8,11,17,28,34,38,39,41,43,46,51,52,53,54,56,64,65,70,72,78\}$ & 709 \\
\hline $\mathrm{x} 40$ & $\{5,6,11,19,27,31,32,39,40,44,47,49,51,52,56,58,59,63,65,66,69,71,79\}$ & 709 \\
\hline $\mathrm{x} 41+1$ & $\{7,9,10,15,17,24,25,26,33,36,43,45,52,56,59,60,61,63,68,71,74,77\}$ & 709 \\
\hline $\mathrm{x} 42+1$ & $\{8,10,11,16,18,25,26,27,34,37,44,46,53,57,60,61,62,64,69,72,75,78\}$ & 710 \\
\hline $\mathrm{x} 43+1$ & $\{9,11,12,17,19,26,27,28,35,38,45,47,54,58,61,62,63,65,70,73,76,79\}$ & 711 \\
\hline $\mathrm{x} 47$ & $\{4,5,7,13,15,18,27,30,33,34,36,39,42,44,45,46,51,53,57,63,75,77,78\}$ & 709 \\
\hline $\mathrm{x} 48+1$ & $\{6,7,15,19,27,30,35,37,44,45,46,47,49,50,56,59,60,67,70,71,72,75,79\}$ & 709 \\
\hline $\mathrm{x} 49$ & $\{0,8,14,18,25,28,31,35,38,42,44,45,51,52,58,60,66,67,70,73,76,77\}$ & 709 \\
\hline $\mathrm{x} 50+1$ & $\{0,2,8,11,14,15,17,21,22,28,31,32,39,41,52,53,59,60,65,67,74,77,78\}$ & 709 \\
\hline $\mathrm{x} 51$ & $\{1,8,10,15,18,26,28,29,33,35,37,38,42,51,53,55,57,60,61,65,66,67,75\}$ & 709 \\
\hline $\mathrm{x} 21+\mathrm{x} 52$ & $\{7,10,11,12,15,21,29,32,37,39,41,44,47,53,56,57,59,62,63,66,70,76\}$ & 710 \\
\hline $\mathrm{x} 53+1$ & $\{1,4,8,10,11,12,14,15,19,22,24,29,31,33,39,42,50,52,55,58,60,61,65\}$ & 710 \\
\hline $\mathrm{x} 23+\mathrm{x} 54+1$ & $\{9,12,13,14,17,23,31,34,39,41,43,46,49,55,58,59,61,64,65,68,72,78\}$ & 712 \\
\hline $\mathrm{x} 24+\mathrm{x} 55+1$ & $\{10,13,14,15,18,24,32,35,40,42,44,47,50,56,59,60,62,65,66,69,73,79\}$ & 713 \\
\hline $\mathrm{x} 57+1$ & $\{1,3,6,10,11,14,15,16,23,25,28,35,40,41,42,44,46,52,58,66,68,69,75\}$ & 709 \\
\hline $\mathrm{x} 21+\mathrm{x} 49+\mathrm{x} 58+1$ & $\{8,12,14,19,26,28,30,40,41,42,43,48,50,53,59,62,63,67,71,72,74,79\}$ & 709 \\
\hline $\mathrm{x} 59+1$ & $\{6,14,16,31,37,40,43,48,50,53,54,55,57,58,60,61,62,68,72,73,74,76\}$ & 709 \\
\hline $\mathrm{x} 60+1$ & $\{3,4,14,16,26,29,30,38,40,43,47,54,56,58,60,64,65,67,69,70,75,76,77\}$ & 709 \\
\hline $\mathrm{x} 61$ & $\{3,8,11,14,16,17,18,20,22,24,27,33,35,38,44,48,52,53,59,66,73,77\}$ & 711 \\
\hline $\mathrm{x} 62$ & $\{4,9,12,15,17,18,19,21,23,25,28,34,36,39,45,49,53,54,60,67,74,78\}$ & 712 \\
\hline $\mathrm{x} 19+\mathrm{x} 63+1$ & $\{2,5,9,17,21,27,28,30,35,37,46,48,50,53,54,60,61,63,65,69,71,73,79\}$ & 709 \\
\hline $\mathrm{x} 67$ & $\{1,7,12,15,18,27,30,41,44,46,47,48,49,52,53,54,56,59,62,63,66,69,79\}$ & 709 \\
\hline $\mathrm{x} 72$ & $\{6,11,16,19,26,34,36,39,41,42,47,49,52,54,57,59,66,67,71,72,76,79\}$ & 709 \\
\hline $\mathrm{x} 73$ & $\{1,3,4,6,12,14,15,19,25,26,28,29,35,40,49,52,57,64,66,67,68,72,75\}$ & 709 \\
\hline $\mathrm{x} 74$ & $\{2,4,5,7,13,15,16,20,26,27,29,30,36,41,50,53,58,65,67,68,69,73,76\}$ & 710 \\
\hline $\mathrm{x} 75$ & $\{3,5,6,8,14,16,17,21,27,28,30,31,37,42,51,54,59,66,68,69,70,74,77\}$ & 711 \\
\hline $\mathrm{x} 76$ & $\{4,6,7,9,15,17,18,22,28,29,31,32,38,43,52,55,60,67,69,70,71,75,78\}$ & 712 \\
\hline
\end{tabular}


After collecting linear expressions and the corresponding cubes we performed the on line stage of cube attack. We chose a random 80-bit key (the secret in our experiment). Then we summed up the ciphertext bits obtained for the chosen cubes and the secret key, where the bits of initial vectors beyond the cube indices were equal to zero. This way we got the exact values of the terms from Table 1 and we obtained the system of linear equations

$$
\begin{aligned}
& x 14=1 \quad x 15=0 \quad x 16=0 \\
& x 17=0 \quad x 18=0 \quad x 19=1 \\
& x 20=1 \quad x 21=0 \quad x 22=1 \\
& x 23=0 \quad x 24=0 \quad x 25=1 \\
& x 33=1 \quad x 35=0 \quad x 39=1 \\
& x 40=1 \quad x 41=0 \quad x 42=0 \\
& x 43=1 \quad x 47=1 \quad x 48=1 \\
& x 49=1 \quad x 50=1 \quad x 51=0 \\
& x 21+x 52=0 \quad x 53=0 \quad x 23+x 54=1 \\
& x 24+x 55=0 \quad x 57=1 \quad x 21+x 49+x 58=1 \\
& x 59=1 \quad x 60=1 \quad x 61=0 \\
& x 62=0 \quad x 19+x 63=1 \quad x 67=0 \\
& x 72=1 \quad x 73=0 \quad x 74=0 \\
& x 75=0 \quad x 76=1
\end{aligned}
$$

The equations $(1) \div(14)$ give the values of 41 bits of the key:

$$
\begin{gathered}
x 14, x 15, x 16, x 17, x 18, x 19, x 20, x 21, x 22, x 23, x 24, x 25, x 33, x 35, \\
x 39, x 40, x 41, x 42, x 43, x 47, x 48, x 49, x 50, x 51, x 52, x 53, x 54, x 55, \\
x 57, x 58, x 59, x 60, x 61, x 62, x 63, x 67, x 72, x 73, x 74, x 75, x 76 .
\end{gathered}
$$

It remained to find 39 bits of the secret key.

\subsection{Quadratic Expressions}

In the preprocessing stage we also found the following cubes with corresponding quadratic terms (Tables 1 and 2). In fact, during this stage, 80 quadraticity tests were executed first, and if the function passed them, the linear tests were applied to check its affineness; additionaly it was checked whether the function was non-constant. The same method was applied to find some cubes with quadratic terms from similar ones by increasing indices and initialization rounds; then the corresponding quadratic polynomials have some regular structure. Having terms from Table 2, the on line stage of the 
cube attack was done with the same secret key obtaining this way the values of these terms.

Table 2. Quadratic expressions.

\begin{tabular}{|c|c|c|}
\hline expression & cube indices & round \\
\hline$\overline{\mathrm{x} 25 \mathrm{x} 26+\mathrm{x} 24+\mathrm{x} 51}$ & $\begin{array}{l}\{0,4,6,13,18,19,22,27,35,37,38,43,46,48,51,53,55,57,60,61,64,66,79\} \\
\end{array}$ & $\overline{709}$ \\
\hline $\mathrm{x} 31 \mathrm{x} 32+\mathrm{x} 30+\mathrm{x} 57$ & $\{3,10,11,24,25,30,34,38,40,41,43,44,51,54,57,59,61,62,65,66,70,76,79\}$ & 709 \\
\hline $\mathrm{x} 32 \times 33+\mathrm{x} 31+\mathrm{x} 58$ & $\{1,6,8,10,13,15,19,20,26,37,39,40,43,47,53,54,57,59,64,67,68,72,75\}$ & 709 \\
\hline $\mathrm{x} 33 \times 34+\mathrm{x} 32+\mathrm{x} 59$ & $\{1,3,10,15,16,18,24,26,28,29,33,37,39,40,43,46,49,51,52,54,59,61,78\}$ & 709 \\
\hline $\mathrm{x} 35 \times 36+\mathrm{x} 34+\mathrm{x} 61$ & $\{1,8,13,16,21,26,27,28,29,31,36,39,47,48,50,56,57,59,60,61,66,72,78\}$ & 709 \\
\hline $\mathrm{x} 37 \times 38+\mathrm{x} 36+\mathrm{x} 63$ & $\{0,1,8,9,10,12,14,18,21,22,23,26,32,33,40,49,52,54,57,67,75,78,79\}$ & 709 \\
\hline $\mathrm{x} 38 \times 39+\mathrm{x} 37+\mathrm{x} 64$ & $\{2,6,14,24,27,29,30,32,38,43,45,46,49,50,53,54,58,67,68,70,74,76,77\}$ & 709 \\
\hline $\mathrm{x} 39 \times 40+\mathrm{x} 38+\mathrm{x} 65$ & $\{0,3,4,11,13,18,20,32,36,43,48,49,52,56,59,63,64,66,67,71,73,76,78\}$ & 709 \\
\hline $\mathrm{x} 18 \mathrm{x} 19+\mathrm{x} 17+\mathrm{x} 44$ & $\{1,9,13,14,16,18,19,31,37,39,40,42,45,46,49,52,53,58,67,68,73,76,78\}$ & 709 \\
\hline $\begin{array}{c}\mathrm{x} 19 \mathrm{x} 20+\mathrm{x} 18+\mathrm{x} 45 \\
+\mathrm{x} 58+1\end{array}$ & $\{0,8,13,14,17,19,20,21,22,24,30,32,39,44,47,52,55,59,60,66,68,77,78\}$ & 709 \\
\hline $\begin{array}{l}\mathrm{x} 47 \mathrm{x} 48+\mathrm{x} 19+\mathrm{x} 40 \\
+\mathrm{x} 46+\mathrm{x} 58+\mathrm{x} 73+1\end{array}$ & $\{0,6,10,15,17,18,20,27,30,31,41,49,54,55,56,58,60,61,65,66,71,74,78\}$ & 709 \\
\hline $\mathrm{x} 64 \mathrm{x} 65+\mathrm{x} 21+\mathrm{x} 63$ & $\{2,4,5,7,10,13,14,16,23,25,28,33,36,42,43,56,59,61,63,68,74,76,79\}$ & 709 \\
\hline $\begin{array}{c}\mathrm{x} 66 \mathrm{x} 67+\mathrm{x} 14+\mathrm{x} 23 \\
+\mathrm{x} 65\end{array}$ & $\{1,6,11,13,15,16,20,21,26,29,30,37,43,46,47,51,55,57,60,69,76,77,79\}$ & 709 \\
\hline $\mathrm{x} 40 \mathrm{x} 41+\mathrm{x} 39+\mathrm{x} 66$ & $\{3,8,12,14,22,24,26,30,32,36,43,44,45,46,49,51,52,53,57,59,72,75,78\}$ & 709 \\
\hline $\begin{array}{c}\mathrm{x} 68 \mathrm{x} 69+\mathrm{x} 16+\mathrm{x} 25 \\
+\mathrm{x} 67+1\end{array}$ & $\{1,3,7,9,11,13,17,20,23,32,38,39,40,44,46,59,62,64,68,70,73,76,78\}$ & 709 \\
\hline $\begin{array}{c}\mathrm{x} 69 \mathrm{x} 70+\mathrm{x} 17+\mathrm{x} 26 \\
+\mathrm{x} 68 \\
\end{array}$ & $\{0,1,6,7,8,13,16,17,18,25,26,33,37,41,49,56,58,61,62,68,71,78,79\}$ & 709 \\
\hline $\mathrm{x} 51 \mathrm{x} 52+\mathrm{x} 50+\mathrm{x} 77$ & $\{1,10,11,14,16,22,24,25,27,41,42,50,51,52,53,54,58,66,71,74,76,78\}$ & 709 \\
\hline $\mathrm{x} 52 \mathrm{x} 53+\mathrm{x} 51+\mathrm{x} 78+1$ & $\{0,1,7,13,15,18,21,29,30,32,39,42,47,48,55,57,63,65,66,67,72,76,77\}$ & 709 \\
\hline $\mathrm{x} 53 \times 54+\mathrm{x} 52+\mathrm{x} 79$ & $\{5,7,9,15,17,18,27,30,32,37,38,44,47,49,50,52,57,66,68,69,74,77,78\}$ & 709 \\
\hline $\mathrm{x} 54 \times 55+\mathrm{x} 11+\mathrm{x} 53$ & $\{2,11,13,16,25,31,36,39,42,46,47,55,58,63,65,67,68,69,70,73,75,76,77\}$ & 710 \\
\hline $\mathrm{x} 55 \times 566+\mathrm{x} 12+\mathrm{x} 54+1$ & $\{3,12,14,17,26,32,37,40,43,47,48,56,59,64,66,68,69,70,71,74,76,77,78\}$ & 711 \\
\hline $\mathrm{x} 56 \mathrm{x} 57+\mathrm{x} 13+\mathrm{x} 55+1$ & $\{4,13,15,18,27,33,38,41,44,48,49,57,60,65,67,69,70,71,72,75,77,78,79\}$ & 712 \\
\hline
\end{tabular}

We got the following quadratic equations:

$$
\begin{aligned}
x 25 x 26+x 24+x 51 & =0 \\
x 31 x 32+x 30+x 57 & =1 \\
x 32 x 33+x 31+x 58 & =0 \\
x 33 x 34+x 32+x 59 & =0 \\
x 35 x 36+x 34+x 61 & =0 \\
x 37 x 38+x 36+x 63 & =1 \\
x 38 x 39+x 37+x 64 & =0 \\
x 39 x 40+x 38+x 65 & =0 \\
x 18 x 19+x 17+x 44 & =0 \\
x 19 x 20+x 18+x 45+x 58 & =1 \\
x 47 x 48+x 19+x 40+x 46+x 58+x 73 & =0 \\
x 64 x 65+x 21+x 63 & =0 \\
x 66 x 67+x 14+x 23+x 65 & =0
\end{aligned}
$$




$$
\begin{aligned}
x 40 x 41+x 39+x 66 & =1 \\
x 68 x 69+x 16+x 25+x 67 & =0 \\
x 69 x 70+x 17+x 26+x 68 & =0 \\
x 51 x 52+x 50+x 77 & =0 \\
x 52 x 53+x 51+x 78 & =0 \\
x 53 x 54+x 52+x 79 & =1 \\
x 54 x 55+x 11+x 53 & =1 \\
x 55 x 56+x 12+x 54 & =1 \\
x 56 x 57+x 13+x 55 & =0
\end{aligned}
$$

We substituted the 41 known key bits to equations $(15) \div(36)$ to obtain new 22 values of key bits:

\begin{tabular}{|c|c|c|}
\hline expression & cube indices & round \\
\hline $\mathrm{x} 8 \mathrm{x} 9+\mathrm{x} 7+\mathrm{x} 34$ & 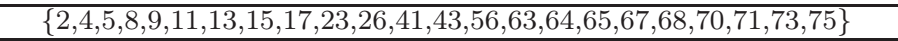 & $\overline{709}$ \\
\hline $\mathrm{x} 7 \mathrm{x} 8+\mathrm{x} 6+\mathrm{x} 33$ & $\{1,4,8,9,16,22,25,28,29,34,42,48,49,53,55,57,59,62,70,73,75,76,79\}$ & 709 \\
\hline $\mathrm{x} 9 \mathrm{x} 10+\mathrm{x} 8+\mathrm{x} 35$ & $\{3,5,6,9,10,12,14,16,18,24,27,42,44,57,64,65,66,68,69,71,72,74,76\}$ & 710 \\
\hline $\mathrm{x} 10 \mathrm{x} 11+\mathrm{x} 9+\mathrm{x} 36$ & $\{4,6,7,10,11,13,15,17,19,25,28,43,45,58,65,66,67,69,70,72,73,75,77\}$ & 711 \\
\hline $\mathrm{x} 11 \mathrm{x} 12+\mathrm{x} 10+\mathrm{x} 37$ & $\{5,7,8,11,12,14,16,18,20,26,29,44,46,59,66,67,68,70,71,73,74,76,78\}$ & 712 \\
\hline $\mathrm{x} 70 \mathrm{x} 71+\mathrm{x} 27+\mathrm{x} 69$ & $\{0,2,5,8,13,14,16,17,27,35,38,43,50,52,53,56,57,60,65,69,75,78,79\}$ & 709 \\
\hline $\mathrm{x} 6 \mathrm{x} 7+\mathrm{x} 5+\mathrm{x} 32$ & $\{0,3,6,9,13,15,16,21,27,33,40,41,43,47,53,57,58,59,61,70,71,76,78\}$ & 709 \\
\hline $\mathrm{x} 4 \mathrm{x} 5+\mathrm{x} 3+\mathrm{x} 30$ & $\{1,4,9,13,14,16,19,24,33,37,38,43,44,45,46,47,54,56,59,68,70,73,76\}$ & 709 \\
\hline $\mathrm{x} 3 \mathrm{x} 4+\mathrm{x} 2+\mathrm{x} 29$ & $\{0,1,2,5,8,9,14,16,25,30,31,40,47,53,54,58,60,61,63,69,75,77,78\}$ & 709 \\
\hline $\mathrm{x} 30 \mathrm{x} 31+\mathrm{x} 29+\mathrm{x} 56$ & $\{8,11,15,20,23,24,27,31,32,36,42,45,50,52,53,54,56,59,60,61,62,68,75\}$ & 709 \\
\hline $\mathrm{x} 5 \mathrm{x} 6+\mathrm{x} 4+\mathrm{x} 31$ & $\{0,5,7,8,9,12,20,25,31,35,42,45,46,47,51,56,58,60,67,68,69,70,71\}$ & 709 \\
\hline $\mathrm{x} 1 \mathrm{x} 2+\mathrm{x} 0+\mathrm{x} 27+1$ & $\{7,8,11,14,15,31,36,37,40,41,44,48,49,55,58,59,62,64,66,67,69,75,78\}$ & 709 \\
\hline $\mathrm{x} 2 \mathrm{x} 3+\mathrm{x} 1+\mathrm{x} 28$ & $\{1,2,6,7,8,10,13,15,23,27,30,32,35,48,49,50,52,53,56,58,68,69,70\}$ & 709 \\
\hline $\begin{array}{c}\mathrm{x} 7 \mathrm{x} 8+\mathrm{x} 16 \mathrm{x} 53+\mathrm{x} 19 \mathrm{x} 20 \\
+\mathrm{x} 6+\mathrm{x} 27+\mathrm{x} 33+1\end{array}$ & $\{0,2,5,19,20,22,28,29,31,36,37,39,40,50,54,55,65,68,71,72,75,77,79\}$ & 710 \\
\hline $\mathrm{x} 71 \mathrm{x} 72+\mathrm{x} 19+\mathrm{x} 28+\mathrm{x} 70$ & $\{0,2,6,9,11,17,21,36,37,42,45,46,47,48,51,53,57,58,60,71,75,77,78\}$ & 710 \\
\hline $\mathrm{x} 29 \times 30+\mathrm{x} 28+\mathrm{x} 55$ & $\{10,14,16,19,24,25,27,32,34,35,37,38,40,42,45,49,52,60,63,70,71,76,78\}$ & 710 \\
\hline
\end{tabular}

$$
\begin{gathered}
x 11=1, x 12=0, x 26=0, x 30=1, x 31=1, x 32=1, x 34=0, x 36=1 \\
x 37=0, x 38=0, x 44=0, x 45=0, x 46=1, x 64=0, x 65=1, \\
x 66=0, x 68=1, x 69=1, x 70=1, x 77=1, x 78=0, x 79=1 .
\end{gathered}
$$

The equation (29) takes the form $x 68 x 69=1$ and the equation (36) gives the relation $x 56=x 13$. At this moment we were left with the remaining 17 unknown key bits:

$$
x 0, \ldots, x 10, x 13, x 27, x 28, x 29, x 56, x 71 .
$$

These key bits were found by considering the following cubes and corresponding quadratic expressions.

Table 3. Quadratic expressions. 
The results of the on line stage of attack led us to the second system of quadratic equations:

$$
\begin{array}{rlrl}
x 8 x 9+x 7=1 & x 7 x 8+x 6=0 \\
x 9 x 10+x 8=1 & x 9+x 10=0 \\
x 10=1 & x 27+x 71=0 \\
x 6 x 7+x 5=0 & x 4 x 5+x 3=1 \\
x 3 x 4+x 2+x 29=0 & x 29+x 56=1 \\
x 5 x 6+x 4=0 & x 1 x 2+x 0+x 27=1 \\
x 2 x 3+x 1+x 28=1 & x 7 x 8+x 6+x 27=0 \\
x 28+x 71=0 & x 28+x 29=1
\end{array}
$$

The above equations after solving them (by hand) gave the following key bits:

$$
\begin{array}{rlll}
x 0=1 & x 1=0 & & x 2=1 \\
x 3=1 & x 4=0 & x 5=0 \\
x 6=0 & x 7=1 & x 8=0 \\
x 9=1 & x 10=1 & x 27=0 \\
x 28=0 & x 29=1 & x 56=0 \\
x 71=0 & &
\end{array}
$$

Finally, we got $x 13=0$, since $x 13=x 56$. These are all key bits:

$$
\begin{gathered}
{[x 0, \ldots, x 79]=} \\
{[1,0,1,1,0,0,0,1,0,1,1,1,0,0,1,0,0,0,0,1,1,0,1,0,0,1,0,0,0,1,1,} \\
1,1,1,0,0,1,0,0,1,1,0,0,1,0,0,1,1,1,1,1,0,0,0,1,0,0,1,0,1,1,0, \\
0,0,0,1,0,0,1,1,1,0,1,0,0,0,1,1,0,1]
\end{gathered}
$$

We would like to indicate that, in the above cube attack with quadraticity tests on a variant of Trivium reduced to 709 initialization rounds, the resulting system of quadratic equations was fairly simple. The method of solving it in two steps enabled us to find solutions just using only some elementary tricks. We have chosen this number of initialization rounds because a reduced variant of Trivium (called Bivium) having only two registers and $4 \times 177=708$ initialization rounds was previously investigated. In an attack on Trivium with more initialization rounds and using quadraticity tests to recover more key bits than only those from linear tests requires more time or computational resources. 


\section{References}

[1] N. Alon, T. Kaufman, M. Krivelevich, S. Litsyn, and D. Ron. Testing Low-Degree Polynomials over GF(2). RANDOM 2003 and APPROX 2003, S. Arora, K. Jansen, J.D.P. Rolim, and A. Sahai, editors. LNCS, vol 2764, pp. 188-199. Springer 2003.

[2] J-P. Aumasson, W. Meier, I. Dinur, and A. Shamir. Cube Testers and Key Recovery Attacks on Reduced Round MD6 and Trivium. Fast Software Encryption 2009. Orr Dunkelman, editor. LNCS, vol 5665, pp. 1-22. Springer 2009.

[3] J-P. Aumasson, I. Dinur, L. Henzen, W. Meier, and A. Shamir. Efficient FPGA Implementations of High-Dimensional Cube Testers on the Stream Cipher Grain-128. IACR Cryptology ePrint Archive, 2009/218.

[4] S. S. Bedi and R. Pillai. Cube Attacks on Trivium. IACR Cryptology ePrint Archive. 2009/15.

[5] M. Blum, M. Luby, and R. Rubinfeld. Self-Testing/Correcting with Applications to Numerical Problems. Journal of Computer and System Sciences, vol 47(1993), pp. 549-595.

[6] Ch. DeCannière and B. Preneel. Trivium. M.J.B. Robshaw and O. Bilet, editors. New Stream Cipher Designs. LNCS, vol 4817, pp. 244-246. Springer 2008.

[7] P. Crowley, Trivium, SSE2, CorePy, and the "cube attack". Published on http://www.lshift.net/blog/

[8] I. Dinur and A. Shamir. Cubic Attacks on Tweakable Black Box Polynomials. EUROCRYPT 2009. A. Joux, editor. LNCS, vol 5479, pp. 278-299. Springer 2009.

[9] I. Dinur and A. Shamir. Side Channel Cube Attacks on Block Ciphers. IACR Cryptology ePrint Archive, 2009/127.

[10] P. Mroczkowski and J. Szmidt. The Cube Attack on Courtois Toy Cipher. IACR Cryptology ePrint Archive, 2009/497. To appear in Proceedings of WEWoRC 2009. LNCS. Springer.

[11] P. Mroczkowski and J. Szmidt. The Cube Attack on Stream Cipher Trivium and Quadraticity Tests. Rump Session. CRYPTO 2010.

[12] M. Vielhaber, Breaking ONE.TRIVIUM by AIDA an Algebraic IV Differential Attack. IACR Cryptology ePrint Archive, 2007/413.

[13] M. Vielhaber. AIDA Breaks (BIVIUM A and B) in 1 Minute Dual Core CPU Time. IACR Cryptology ePrint Archive, 2009/402. 